\title{
RUIN PROBABILITY FOR THE INTEGRATED GAUSSIAN PROCESS WITH FORCE OF INTEREST
}

\author{
XIAOXIA HE* AND \\ YIJUN HU, ${ }^{* *}$ Wuhan University
}

\begin{abstract}
In this paper we obtain the exact asymptotics of the ruin probability for the integrated Gaussian process with force of interest. The results obtained are consistent with those obtained for the case in which there is no force of interest.

Keywords: Integrated Gaussian process; ruin probability; Rice method; force of interest

2000 Mathematics Subject Classification: Primary 60G15

Secondary $60 \mathrm{G} 70$
\end{abstract}

\section{Introduction and main result}

In risk analysis of insurance and finance, ruin is the most important event because it should be avoided. The classical risk model is defined as

$$
U(t)=u+c t-\sum_{k=1}^{N(t)} X_{k},
$$

where $N=\{N(t) ; t \geq 0\}, N(0)=0$ is a point process, and $\left\{X_{k}\right\}_{k=1}^{\infty}$ is a sequence of identically distributed random variables. The sum in the risk process represents the total claim amount up to time $t$ and the positive constant $c$ is the premium rate. For such a risk process, the probability of ruin, the ruin time, the distribution of the surplus, and some related quantities were investigated by Dufresne and Gerber [3], Dickson and Egídio dos Reis [2], Rolski et al. [10, pp. 147-204], etc.

If we consider a large company with many customers, the random walk $\sum_{k=1}^{N(t)} X_{k}$ with random times is replaced by another random process $X(t)$, such as a Brownian motion, a Lévy process, or a renewal process. In the latter cases, the ruin probability is still of importance. Thus, the process $X(t)$ has been of interest in such models as the Brownian motion, the fractional Brownian motion, and the integrated Gaussian process, see [1], [6], and [8].

In [7], the following model was considered:

$$
Y_{t}=\int_{0}^{t} \xi(s) \mathrm{d} s-c t,
$$

where $\xi(t)$ is a stationary, real-valued, and zero-mean Gaussian process with a real twice differential covariance function $R(t)$, and $c>0$ is a constant. Such a model arises in ruin

Received 8 January 2007; revision received 11 July 2007.

* Current address: College of Science, Wuhan University of Science and Technology, Wuhan, 430081, P. R. China.

Email address: elizabeth_he@163.com

** Postal address: School of Mathematics and Statistics, Wuhan University, Wuhan, 430072, P. R. China.

Email address: yijunhu@public.wh.hb.cn 
problems in finance, information transfer, and storage problems [6]. The exact asymptotics of the ruin probability was found by Kobelkov [7] using Rice's method.

In the present paper we assume that there exists a constant force of interest $\delta>0$ which affects the risk process. Let $u>0$ be the initial surplus of the insurance company, then the total surplus up to time $t$, denoted by $U_{\delta}(t)$, satisfies the equation

$$
U_{\delta}(t)=u \mathrm{e}^{\delta t}+c \int_{0}^{t} \mathrm{e}^{\delta z} \mathrm{~d} z-\int_{0}^{t} \mathrm{e}^{\delta(t-z)} \xi(z) \mathrm{d} z .
$$

The ultimate ruin probability for this risk process is then defined by

$$
\mathcal{P}(u)=\mathrm{P}\left\{U_{\delta}(t)<0 \text { for some } t \geq 0\right\}=\mathrm{P}\left\{\max _{t \geq 0} U_{\delta}(t)<0\right\} .
$$

The aim of this paper is to obtain the exact asymptotics of the ruin probability $\mathcal{P}(u)$ as $u \rightarrow \infty$. To evaluate the asymptotic behavior of the ruin probability (1.1), we transform the probability in the following way:

$$
\begin{aligned}
\mathcal{P}(u) & =\mathrm{P}\left\{\max _{t \geq 0} \int_{0}^{t} \mathrm{e}^{-\delta z} \xi(z) \mathrm{d} z>u+c \int_{0}^{t} \mathrm{e}^{-\delta z} \mathrm{~d} z\right\} \\
& =\mathrm{P}\left\{\max _{t \geq 0} \frac{1}{1+(c / u) \int_{0}^{t} \mathrm{e}^{-\delta z} \mathrm{~d} z} \int_{0}^{t} \mathrm{e}^{-\delta z} \xi(z) \mathrm{d} z>u\right\} \\
& =\mathrm{P}\left\{\max _{t \geq 0} V_{t}>u\right\}
\end{aligned}
$$

where

$$
V_{t}=\frac{1}{1+(c / u) \int_{0}^{t} \mathrm{e}^{-\delta z} \mathrm{~d} z} \int_{0}^{t} \mathrm{e}^{-\delta z} \xi(z) \mathrm{d} z
$$

Now, we are in a position to state our main result.

Theorem 1.1. Assume that $R(t)$ is the covariance function for the real-valued and zeromean stationary Gaussian process $\xi(t), R(t)$ is twice differentiable for any $t$, and that $G=$ $\int_{0}^{\infty} \mathrm{e}^{-\delta z} R(z) \mathrm{d} z<\infty$ and $H=\int_{0}^{\infty} \mathrm{e}^{\delta z} R(z) \mathrm{d} z<\infty$. Then we have

$$
\mathcal{P}(u)=\frac{1}{\sqrt{2 \pi} \delta} \log \left(\frac{(\delta u+c) H}{G c}\right) \exp \left(-\frac{u^{2}}{2 G}\left(1+\frac{c}{u}\left(1-\frac{G c}{\delta(\delta u+c) H}\right)\right)^{2}\right)(1+o(1)) .
$$

Remark. The covariance function $R(t)$ is usually assumed to be regularly varying; see, for example, [1] and [5]. However, the regularly varying property of $R(t)$ does not satisfy the condition in our theorem. But, there exists an example which meets the conditions of Theorem 1.1. For example, when $R(t)=c \exp \left(-|t|^{2} / 2\right), c>0$, it is easy to show that $\int_{0}^{\infty} \mathrm{e}^{\delta z} R(z) \mathrm{d} z<\infty$. Meanwhile, it does not satisfy the condition of [1] and [5].

To estimate the ruin probability, we shall use the Rice method based on an estimation of the moment of the number of crossings of the level $u$ by the process. Intuitively, it is clear that for large $u$ the event that the process $V_{t}$ crosses the level $u$ more than once is rare, so the probability $\mathcal{P}(u)$ approximately equals the mean number of crossings.

Denote by $N_{u}([0, T])$ the number of crossings of the level $u$ by the process $V_{t}$ on the segment $[0, T]$. In [8] and [9], it was shown that, for a random process with continuously differentiable 
trajectories and for any segment $S$, the following inequality holds:

$$
0 \leq \mathrm{E} N_{u}(S)-\mathrm{P}\left\{\max _{t \in S} V_{t} \geq u\right\} \leq \frac{1}{2} \mathrm{E}\left(N_{u}(S)\left(N_{u}(S)-1\right)\right) .
$$

In the next section we will prove Theorem 1.1 in three steps. Firstly, we will obtain asymptotics of the value $\mathrm{E} N_{u}([0, \infty))$ as $u \rightarrow \infty$. Secondly, we will show that, for some $\alpha=\alpha(u)$ and $\beta=\beta(u), \alpha<\beta$,

$$
\mathrm{P}\left\{\max _{t \in[0, \alpha]} V_{t}>u\right\}+\mathrm{P}\left\{\max _{t \in[\beta, \infty]} V_{t}>u\right\}=o\left(\mathrm{E} N_{u}([0, \infty))\right), \quad u \rightarrow \infty .
$$

Finally, we will show that $\mathrm{E}\left(N_{u}([\alpha, \beta])\left(N_{u}([\alpha, \beta])-1\right)\right)=o\left(\mathrm{E} N_{u}([0, \infty))\right)$. Consequently, by inequality (1.2), it follows that

$$
\mathcal{P}(u)=\mathrm{E} N_{u}([0, \infty))(1+o(1)), \quad u \rightarrow \infty .
$$

\section{Proof of Theorem 1.1}

\subsection{Estimation of $\mathbf{E} N_{u}([0, \infty))$}

By the Rice formula, we have

$$
\operatorname{E} N_{u}([0, T])=\int_{0}^{T} \int_{0}^{\infty}|y| p_{t}(u, y) \mathrm{d} y \mathrm{~d} t
$$

where $p_{t}(u, y)$ is a joint distribution density of the random variables $V_{t}$ and $V_{t}^{\prime}$; here and in the sequel, $V_{t}^{\prime}$ denotes the derivative of $V_{t}$. Introduce the notation $a(t)=\operatorname{cov}\left(V_{t}, V_{t}\right)$, $b(t)=\operatorname{cov}\left(V_{t}, V_{t}^{\prime}\right), d(t)=\operatorname{cov}\left(V_{t}^{\prime}, V_{t}^{\prime}\right)$, and $\sigma(t)=\sqrt{a(t) d(t)-b^{2}(t)}$. It is easy to show that

$$
\begin{aligned}
a(t)= & \frac{\int_{0}^{t} \mathrm{e}^{-\delta z} R(z) \mathrm{d} z-\mathrm{e}^{-2 \delta t} \int_{0}^{t} \mathrm{e}^{\delta z} R(z) \mathrm{d} z}{\left(1+(c / u) \int_{0}^{t} \mathrm{e}^{-\delta z} \mathrm{~d} z\right)^{2}}, \\
b(t)= & \frac{\mathrm{e}^{-2 \delta t}}{\left(1+(c / u) \int_{0}^{t} \mathrm{e}^{-\delta z} \mathrm{~d} z\right)^{2}} \int_{0}^{t} \mathrm{e}^{\delta z} R(z) \mathrm{d} z \\
& -\frac{(c / u) \mathrm{e}^{-\delta t}}{\left(1+(c / u) \int_{0}^{t} \mathrm{e}^{-\delta z} \mathrm{~d} z\right)^{3}}\left(\int_{0}^{t} \mathrm{e}^{-\delta z} R(z) \mathrm{d} z-\mathrm{e}^{-2 \delta t} \int_{0}^{t} \mathrm{e}^{\delta z} R(z) \mathrm{d} z\right), \\
d(t)= & \frac{\mathrm{e}^{-\delta t} R(0)}{\left(1+(c / u) \int_{0}^{t} \mathrm{e}^{-\delta z} \mathrm{~d} z\right)^{2}} \\
& +\frac{\left(c^{2} / u^{2}\right) \mathrm{e}^{-2 \delta t}}{\left(1+(c / u) \int_{0}^{t} \mathrm{e}^{-\delta z} \mathrm{~d} z\right)^{4}}\left(\int_{0}^{t} \mathrm{e}^{-\delta z} R(z) \mathrm{d} z-\mathrm{e}^{-2 \delta t} \int_{0}^{t} \mathrm{e}^{\delta z} R(z) \mathrm{d} z\right) \\
& -\frac{(c / u) \mathrm{e}^{-3 \delta t}}{\left(1+(c / u) \int_{0}^{t} \mathrm{e}^{-\delta z} \mathrm{~d} z\right)^{3}} \int_{0}^{t} \mathrm{e}^{\delta z} R(z) \mathrm{d} z .
\end{aligned}
$$

We will prove the finiteness of the following limit:

$$
I \equiv \lim _{T \rightarrow \infty} \operatorname{E} N_{u}([0, T])=\int_{0}^{\infty} \int_{0}^{\infty} \frac{y}{2 \pi \sigma(t)} \exp \left(-\frac{f(u, t)}{\sigma^{2}(t)}\right) \mathrm{d} y \mathrm{~d} t,
$$


where

$$
f(u, t)=d(t) u^{2}-2 b(t) u y+a(t) y^{2} .
$$

Write $\gamma=\gamma(t, u)=-u b(t) /(\sigma(t) \sqrt{a(t)})$. Noting that

$$
\begin{aligned}
\int_{0}^{\infty} y p_{t}(u, y) \mathrm{d} y= & \frac{1}{2 \pi \sigma(t)} \exp \left(-\frac{u^{2}}{2 a(t)}\right) \\
& \times \int_{0}^{\infty} \frac{y}{2 \pi \sigma(t)} \exp \left(-\frac{1}{2 \sigma^{2}(t)}\left(\sqrt{a(t)} y-\frac{u b(t)}{\sqrt{a(t)}}\right)^{2}\right) \mathrm{d} y
\end{aligned}
$$

we have

$$
I=\frac{1}{\sqrt{2 \pi}} \int_{0}^{\infty} \frac{\sigma(t)}{a(t)} g(t) \exp \left(-\frac{u^{2}}{2 a(t)}\right) \mathrm{d} t,
$$

where $g(t)=(1 / \sqrt{2 \pi}) \int_{\gamma}^{\infty} y \mathrm{e}^{-y^{2} / 2} \mathrm{~d} y-\gamma(1-\Phi(\gamma))$ and $\Phi(t)$ are the distribution functions of the standard normal random variable.

It is easy to verify that $g(t)$ is a bounded function, $\sigma(t) / a(t) \rightarrow C_{1}$ as $t \rightarrow 0$, where $C_{1}$ is some constant, and that $\sigma(t) / a(t) \rightarrow 0$ as $t \rightarrow \infty$. So, the limit in (2.1) exists for all $u>0$.

Next we shall evaluate the integral in (2.1) as $u \rightarrow \infty$. First, we will give the following estimation of the probabilities:

$$
\mathrm{P}_{\alpha}=\mathrm{P}\left\{\max _{0 \leq t<\alpha} V_{t}>u\right\}, \quad \mathrm{P}_{\beta}=\mathrm{P}\left\{\max _{t \geq \beta} V_{t}>u\right\},
$$

where $\alpha=t_{0}-(\log u) / u^{3}$ and $\beta=t_{0}+(\log u) / u^{3}$.

Owing to the continuous differentiability of the covariance function of the process $V_{t}$, note that, for $u>0$ and any segment $\left[s_{0}, s_{1}\right]$, there exists a constant $M_{1}(S)$ such that $\mathrm{E}\left(V_{t}-V_{s}\right)^{2} \leq$ $M_{1}(S)|t-s|$ for all $t, s \in S$. By the Piterbarg inequality [8], we obtain

$$
\mathrm{P}\left\{\max _{t \in S} V_{t}>u\right\}<C_{2} \max _{t \in S} \operatorname{var}\left(V_{t}\right) \exp \left(-\frac{u^{2}}{2 \max _{t \in S} \operatorname{var}\left(V_{t}\right)}\right),
$$

where $\operatorname{var}\left(V_{t}\right)$ is the variance function of the process $V_{t}$ and the constant $C_{2}$ depends only on $s_{1}-s_{0}$.

Divide the variance of the process $V_{t}$ into two parts as follows:

$$
\begin{aligned}
\operatorname{var}\left(V_{t}\right) & =\frac{\int_{0}^{t} \mathrm{e}^{-\delta z} R(z) \mathrm{d} z-\mathrm{e}^{-2 \delta t} \int_{0}^{t} \mathrm{e}^{\delta z} R(z) \mathrm{d} z}{\left(1+(c / u) \int_{0}^{t} \mathrm{e}^{-\delta z} \mathrm{~d} z\right)^{2}} \\
& =\frac{G-\mathrm{e}^{-2 \delta t} H}{\left(1+(c / u) \int_{0}^{t} \mathrm{e}^{-\delta z} \mathrm{~d} z\right)^{2}}-\frac{\int_{t}^{\infty} \mathrm{e}^{-\delta z} R(z) \mathrm{d} z-\mathrm{e}^{-2 \delta t} \int_{t}^{\infty} \mathrm{e}^{\delta z} R(z) \mathrm{d} z}{\left(1+(c / u) \int_{0}^{t} \mathrm{e}^{-\delta z} \mathrm{~d} z\right)^{2}} \\
& =S_{1}(t)+R_{1}(t),
\end{aligned}
$$

where $G$ and $H$ are as given in Theorem 1.1.

Consider the function $S_{1}(t)$. It reaches its maximum at the point $t_{0}=(1 / \delta) \log ((\delta u+$ c) $H / G c)$. For the second derivative of $S_{1}(t)$, we have

$$
\frac{\partial^{2} S_{1}(t)}{\partial t^{2}} \rightarrow-4 \delta^{2} H \mathrm{e}^{-2 \delta t}<0 \quad \text { as } u \rightarrow \infty
$$


Then, for any $0<\gamma<1$, the second derivative converges uniformly on the segment $\left[\gamma(1 / \delta) \log ((\delta u+c) H / G c), \gamma^{-1}(1 / \delta) \log ((\delta u+c) H / G c)\right]$ to the function $S_{2}(t)$. On this segment it is easy to see that $S_{2}(t)<0$, so, $\partial^{2}\left(S_{1}(t)\right) / \partial t^{2}<0$. Thus, we obtain the uniqueness of the maximum on the segment $\left[\gamma(1 / \delta) \log ((\delta u+c) H / G c), \gamma^{-1}(1 / \delta) \log ((\delta u+c) H / G c)\right]$ for large enough $u$.

To estimate $\mathrm{P}_{\beta}$, let us point out that

$$
\exp \left(-\frac{u^{2}}{2 \operatorname{var}\left(V_{t}\right)}\right)=\exp \left(-\frac{u^{2}}{2 S_{1}(t)}+\frac{u^{2} R_{1}(t)}{2 S_{1}(t)\left(S_{1}(t)+R_{1}(t)\right)}\right) .
$$

Consider $t \in\left[\beta, \gamma^{-1}(1 / \delta) \log ((\delta u+c) H / G c)\right]$, we have, for some $\theta \in\left(t_{0}, t\right)$,

$$
\begin{aligned}
\exp \left(-\frac{u^{2}}{2 \operatorname{var}\left(V_{t}\right)}\right) & \leq C_{3} \exp \left(-\frac{u^{2}}{2 S_{1}(t)}\right) \\
& =C_{3} \exp \left(-\frac{u^{2}}{2 S_{1}\left(t_{0}\right)}+\frac{u^{2} S_{1}^{\prime \prime}(\theta)\left(t-t_{0}\right)^{2} / 2}{2\left(S_{1}\left(t_{0}\right)+S_{1}^{\prime \prime}(\theta)\left(t-t_{0}\right)^{2} / 2\right) S_{1}\left(t_{0}\right)}\right) .
\end{aligned}
$$

By the facts that $S_{1}\left(t_{0}\right)=O(1), S_{1}^{\prime \prime}(\theta)=O\left(u^{-2}\right)$, and $t-t_{0} \leq \beta-(1 / \delta) \log ((\delta u+$ c) $H / G c)$, we obtain

$$
\mathrm{P}_{\beta}=o\left(\exp \left(-\frac{u^{2}}{2 S_{1}\left(t_{0}\right)}\right)\right) .
$$

Let $t^{*}$ denote some point of maximum variance of the random process and let $t_{0}$ denote the maximum point of the function $S_{1}(t)$ in the neighbourhood of $(1 / \delta) \log ((\delta u+c) H / G c)$. Then

$$
\begin{aligned}
\exp \left(-\frac{u^{2}}{2 \max _{0<t \leq \alpha} \operatorname{var}\left(V_{t}\right)}\right) & =\exp \left(\frac{u^{2} R_{1}(t)}{2\left(S_{1}\left(t^{*}\right)+R_{1}\left(t^{*}\right)\right)}\right) \\
& =\exp \left(-\frac{u^{2}}{2\left(S_{1}\left(t_{0}\right)+\left(S_{1}\left(t^{*}\right)+R_{1}\left(t^{*}\right)-S_{1}\left(t_{0}\right)\right)\right)}\right) .
\end{aligned}
$$

Noting the facts that $S_{1}\left(t^{*}\right)-S_{1}\left(t_{0}\right)<0$ and $R_{1}\left(t^{*}\right) \rightarrow 0$, by the same argument used to derive (2.2), we can obtain

$$
\mathrm{P}_{\alpha}=o\left(\exp \left(-\frac{u^{2}}{2 S_{1}\left(t_{0}\right)}\right)\right)
$$

Next, we turn to evaluating the integral

$$
I=\frac{1}{\sqrt{2 \pi}} \int_{\alpha}^{\beta} \frac{\sigma(t)}{a(t)} g(t) \exp \left(-\frac{u^{2}}{2 a(t)}\right) \mathrm{d} t .
$$

Taking a change of variable $t=t(\tau)=(\tau / \delta) \log ((\delta u+c) H / G c)$ gives

$$
I=\frac{1}{\sqrt{2 \pi} \delta} \log \left(\frac{(\delta u+c) H}{G c}\right) \int_{\tilde{\alpha}}^{\tilde{\beta}} \frac{\sigma(t(\tau))}{a(t(\tau))} g(t(\tau)) \exp \left(-\frac{u^{2}}{2 a(t(\tau))}\right) \mathrm{d} \tau,
$$

where

$$
\tilde{\alpha}=\left(\frac{\delta}{\log ((\delta u+c) H / G c)}\right) \quad \text { and } \quad \tilde{\beta}=\left(\frac{\delta}{\log ((\delta u+c) H / G c)}\right) \beta .
$$

Noting that

$$
a(t(\tau))=S_{1}(t(\tau))+R_{1}(t(\tau)),
$$


we have

$$
\frac{u^{2}}{2 a(t(\tau))}=\frac{u^{2}}{2\left(S_{1}(t(\tau))+R_{1}(t(\tau))\right)}=S_{3}(\tau)-\frac{u^{2} R_{1}(t(\tau))}{2 S_{1}(t(\tau))\left(S_{1}(t(\tau))+R_{1}(t(\tau))\right)},
$$

where $S_{3}(\tau)=u^{2} / 2 S_{1}(\tau)$. Thanks to the assumptions, we find that the last term in (2.4) tends to 0 . Thus,

$$
I=\frac{1}{\sqrt{2 \pi} \delta} \log \left(\frac{(\delta u+c) H}{G c}\right) \int_{\tilde{\alpha}}^{\tilde{\beta}} \frac{\sigma(t(\tau))}{a(t(\tau))} g(t(\tau)) \exp \left(-S_{3}(\tau)\right) \mathrm{d} \tau(1+o(1)) .
$$

Since the function $S_{3}(\tau)$ has a unique minimum point $\tau_{\min }$ on the segment $[\alpha, \beta]$, by applying Laplace's method to the integral in (2.5) (see Chapter 3 of Freidlin and Wentzell [4]), we have

$$
I=\frac{1}{\sqrt{2 \pi} \delta} \log \left(\frac{(\delta u+c) H}{G c}\right) \exp \left(-S_{3}\left(\tau_{\min }\right)\right)(1+o(1)) .
$$

Thus,

$$
I=\frac{1}{\sqrt{2 \pi} \delta} \log \left(\frac{(\delta u+c) H}{G c}\right) \exp \left(-\frac{u^{2}}{2 G}\left(1+\frac{c}{u}\left(1-\frac{G c}{\delta(\delta u+c) H}\right)\right)^{2}\right)(1+o(1)) .
$$

\subsection{Estimation of the second factorial moment}

Taking into account (2.2) and (2.3), it remains to estimate the second factorial moment of the number of crossings of the level $u$ by the process $V_{t}$ on the segment $[\alpha, \beta]$. Let

$$
V_{t}=\frac{1}{(1+(c / u)) \int_{0}^{t} \mathrm{e}^{-\delta z} \mathrm{~d} z} \int_{0}^{t} \mathrm{e}^{-\delta z} \xi(z) \mathrm{d} z
$$

then

$$
\mathcal{P}(u)=\mathrm{P}\left\{\max _{t \geq 0} \frac{1}{(1+(c / u)) \int_{0}^{t} \mathrm{e}^{-\delta z} \mathrm{~d} z} \int_{0}^{t} \mathrm{e}^{-\delta z} \xi(z) \mathrm{d} z>u\right\}=\mathrm{P}\left\{\max _{t>0} V_{t}>u\right\} .
$$

By explicit formula for the second factorial moment [8], we have

$$
\mathrm{E}\left(N_{u}([\alpha, \beta])\left(N_{u}([\alpha, \beta])-1\right)\right)=\int_{\alpha}^{\beta} \int_{\alpha}^{\beta} \int_{0}^{\infty} \int_{0}^{\infty} y_{1} y_{2} \varphi_{t, s, t, s}\left(u, u, y_{1}, y_{2}\right) \mathrm{d} y_{1} \mathrm{~d} y_{2} \mathrm{~d} s \mathrm{~d} t,
$$

where $\varphi_{t, s, t, s}\left(u, u, y_{1}, y_{2}\right)$ is a joint density of the variables $V_{t}, V_{s}, V_{t}^{\prime}$, and $V_{s}^{\prime}$. Denoting $\Omega=\{(s, t): x \in[\alpha, \beta], y \in[\alpha, \beta]\}$, then we can obtain

$$
\begin{aligned}
\int_{\alpha}^{\beta} \int_{\alpha}^{\beta} \int_{0}^{\infty} \int_{0}^{\infty} y_{1} y_{2} \varphi_{t, s, t, s}\left(u, u, y_{1}, y_{2}\right) \mathrm{d} y_{1} \mathrm{~d} y_{2} \mathrm{~d} s \mathrm{~d} t \\
\leq \iint_{\Omega \cap(t \geq s)} \mathrm{d} s \mathrm{~d} t \int_{0}^{\infty} \int_{0}^{\infty}\left(y_{1}^{2}+y_{2}^{2}\right) \varphi_{t, s, t, s}\left(u, u, y_{1}, y_{2}\right) \mathrm{d} y_{1} \mathrm{~d} y_{2} \\
\leq \iint_{\Omega \cap(t \geq s)} \mathrm{d} s \mathrm{~d} t \int_{0}^{\infty} y^{2} \varphi_{t, s, t}(u, u, y) \mathrm{d} y \\
\quad+\iint_{\Omega \cap(t \geq s)} \mathrm{d} s \mathrm{~d} t \int_{0}^{\infty} y^{2} \varphi_{t, s, s}(u, u, y) \mathrm{d} y,
\end{aligned}
$$


where $\varphi_{t, s, t}(u, u, y)$ is a joint density of the variables $V_{t}, V_{s}$, and $V_{s}^{\prime}$. Let us estimate the first integral; estimation of the latter one is performed similarly. Proceeding to a conditional density leads to

$$
\begin{aligned}
& \iint_{\Omega \cap(t \geq s)} \mathrm{d} s \mathrm{~d} t \int_{0}^{\infty} y^{2} \varphi_{t, s, t}(u, u, y) \mathrm{d} y \\
& \quad=\iint_{\Omega \cap(t \geq s)} \varphi_{t, s}(u, u) \mathrm{d} s \mathrm{~d} t \int_{0}^{\infty} y^{2} \varphi_{t, s}(y \mid u, u) \mathrm{d} y .
\end{aligned}
$$

Hence, $\varphi_{t, s}(u, u)$ denotes a joint density of the variables $V_{t}$ and $V_{s}$, and $\varphi_{t, s}(y \mid u, u)$ denotes a conditional density of the random variable $V_{t}^{\prime}$ under the conditions that $V_{t}=u$ and $V_{s}=u$. Thus,

$$
\mathrm{E}\left(N_{u}([\alpha, \beta])\left(N_{u}([\alpha, \beta])-1\right)\right) \leq 2 \iint_{\Omega \bigcap(t \geq s)} \varphi_{t, s}(u, u) \mathrm{d} s \mathrm{~d} t \int_{0}^{\infty} y^{2} \varphi_{t, s}(y \mid u, u) \mathrm{d} y .
$$

Since

$$
\begin{gathered}
\varphi_{t, s}(u, u)=\frac{1}{2 \pi \sigma} \exp \left(-\frac{u^{2}}{2 \sigma^{2}}\left(\operatorname{var}\left(V_{s}\right)+\operatorname{var}\left(V_{t}\right)-2 \operatorname{cov}\left(V_{s}, V_{t}\right)\right)\right), \\
\varphi_{t, s}(y \mid u, u)=\frac{1}{\sqrt{2 \pi \Delta}} \exp \left(-\frac{1}{2 \Delta^{2}}(y-m(u, u))^{2}\right),
\end{gathered}
$$

where

$$
\begin{gathered}
\sigma^{2}=\operatorname{var}\left(V_{t}\right) \operatorname{var}\left(V_{s}\right)-\left(\operatorname{cov}\left(V_{t}, V_{s}\right)\right)^{2}, \\
m(u, u)=\frac{u}{\sigma^{2}}\left(\operatorname{var}\left(V_{s}\right) b(t)-\operatorname{cov}\left(V_{s}, V_{t}\right)(b(t)+b(s))+\operatorname{var}\left(V_{t}\right) b(s)\right), \\
b(s)=\operatorname{cov}\left(V_{t}^{\prime}, V_{s}\right), \\
\Delta=\operatorname{var}\left(V_{t}^{\prime}\right)-\frac{\operatorname{var}\left(V_{s}\right) b^{2}(t)-2 \operatorname{cov}\left(V_{t}, V_{s}\right) b(s) b(t)+\operatorname{var}\left(V_{t}\right) b^{2}(s)}{\sigma^{2}},
\end{gathered}
$$

we have

$$
\begin{aligned}
& \mathrm{E}\left(N_{u}([\alpha, \beta])\left(N_{u}([\alpha, \beta])-1\right)\right) \\
& \quad \leq 2(\beta-\alpha)^{2} \sup _{t \geq s} \sigma \varphi_{t, s}(u, u) \sup _{t \geq s} \int_{0}^{\infty} \frac{y^{2}}{\sigma} \varphi_{t, s}(y \mid u, u) \mathrm{d} y .
\end{aligned}
$$

Next we will prove that the former supremum in (2.6) has the order

$$
\log \left(\frac{(\delta u+c) H}{G c}\right) \exp \left(-\frac{u^{2}}{2 G}\left(1+\frac{c}{u}\left(1-\frac{G c}{\delta(\delta u+c) H}\right)\right)^{2}\right)
$$

and that the latter supremum grows no faster than $(\log u) / u^{3}$.

When $t \geq s$, taking a change of variable $y^{\prime}=(y-m) / \Delta$, we have

$$
\int_{0}^{\infty} \frac{y^{2}}{\sigma} \varphi_{t, s}(y \mid u, u) \mathrm{d} y=\int_{-m / \Delta}^{\infty} \frac{(\Delta y+m)^{2}}{\sqrt{2 \pi} \sigma} \exp \left(-\frac{y^{2}}{2}\right) \mathrm{d} y .
$$


Let us state the following facts which will be necessary to estimate the order of $m$ and $\Delta$ :

$$
\begin{gathered}
\operatorname{var}\left(V_{t}\right)=\frac{1}{\left(1+(c / u) \int_{0}^{t} \mathrm{e}^{-\delta z} \mathrm{~d} z\right)^{2}}\left(\int_{0}^{t} \mathrm{e}^{-\delta z} R(z) \mathrm{d} z-\mathrm{e}^{-2 \delta t} \int_{0}^{t} \mathrm{e}^{\delta z} \mathrm{~d} z\right) \\
\operatorname{var}\left(V_{t}^{\prime}\right)=\frac{c \mathrm{e}^{-\delta t}}{\left(1+(c / u) \int_{0}^{t} \mathrm{e}^{-\delta z} \mathrm{~d} z\right)^{2}}+\frac{c(0)}{u\left(1+(c / u) \int_{0}^{t} \mathrm{e}^{-\delta z} \mathrm{~d} z\right)^{3}} \mathrm{e}^{-2 \delta t} \int_{0}^{t} \mathrm{e}^{\delta z} R(z) \mathrm{d} z \\
+\frac{c \mathrm{e}^{-\delta t}\left(\int_{0}^{t} \mathrm{e}^{-\delta z} R(z) \mathrm{d} z-\mathrm{e}^{-2 \delta t} \int_{0}^{t} \mathrm{e}^{\delta z} \mathrm{~d} z\right)}{u\left(1+(c / u) \int_{0}^{t} \mathrm{e}^{-\delta z} \mathrm{~d} z\right)^{4}} \\
\operatorname{cov}\left(V_{t}^{\prime}, V_{s}\right)=\mathrm{e}^{-2 \delta t} \int_{0}^{t-s} \mathrm{e}^{\delta z} R(z) \mathrm{d} z
\end{gathered}
$$

Introduce the functions

$$
f(s)=\operatorname{cov}\left(V_{t}^{\prime}, V_{s}\right), \quad g(s)=\operatorname{var}\left(V_{s}\right), \quad \text { and } \quad h(s)=\operatorname{cov}\left(V_{t}, V_{s}\right) .
$$

Then we have

$$
h^{\prime}(t)=f(t)=\frac{1}{2} g^{\prime}(t), \quad f^{\prime}(t)=\operatorname{var}\left(V_{t}\right), \quad g^{\prime \prime}(t)=2\left(f^{\prime}(t)+h^{\prime \prime}(t)\right) .
$$

Conversely, when $|t-s| \rightarrow 0$, we have

$$
\begin{gathered}
m \sigma^{2}=O\left((s-t)^{3}\right), \\
\Delta^{2} \sigma^{2}=O\left((s-t)^{3}\right), \\
\sigma^{2}=\left(g^{\prime \prime}(\xi) g(t)-2 h(\xi) h^{\prime \prime}(\xi)-2\left(h^{\prime}(\xi)\right)^{2}\right)(s-t)^{2}, \quad \xi \in(s, t) .
\end{gathered}
$$

From (2.6)-(2.10), we have

$$
\begin{gathered}
\sigma^{2} \geq \frac{u^{2} R(0)}{8}(s-t)^{2}, \\
|m| \sigma^{2} \leq C_{4} u^{2}, \quad \Delta \sigma \leq C_{4} u^{2}, \quad C_{4}>0 .
\end{gathered}
$$

Next we will estimate the integral in (2.6). From (2.8)-(2.11), it follows that

$$
\begin{aligned}
\int_{0}^{\infty} \frac{y^{2}}{\sigma} \varphi_{t, s}(y \mid u, u) \mathrm{d} y= & \int_{-m / \Delta}^{\infty} \frac{(\Delta y+m)^{2}}{\sqrt{2 \pi} \sigma} \exp \left(-\frac{y^{2}}{2}\right) \mathrm{d} y \\
= & \frac{\Delta^{2}}{\sqrt{2 \pi} \sigma} \int_{-m / \Delta}^{\infty} y^{2} \exp \left(-\frac{y^{2}}{2}\right) \mathrm{d} y \\
& +\frac{2 m \Delta}{\sqrt{2 \pi} \sigma} \int_{-m / \Delta}^{\infty} y \exp \left(-\frac{y^{2}}{2}\right) \mathrm{d} y \\
& +\frac{m^{2}}{\sqrt{2 \pi} \sigma} \int_{-m / \Delta}^{\infty} \exp \left(-\frac{y^{2}}{2}\right) \mathrm{d} y \\
\leq & C_{5} u .
\end{aligned}
$$

Since $\beta-\alpha \leq 2(\log u) / u^{3}$, we have

$$
2(\beta-\alpha)^{2} \sup _{t \geq s} \int_{o}^{\infty} \frac{y^{2}}{\sigma} \varphi_{t, s}(u, u) \mathrm{d} y \rightarrow 0 \quad \text { as } u \rightarrow \infty .
$$


Therefore, all that remains is to prove that

$$
\begin{aligned}
\sup _{t \geq s} \sigma \varphi_{t, s}(u, u)=o & \left(\log \left(\frac{(\delta u+c) H}{G c}\right)\right. \\
& \left.\times \exp \left(-\frac{u^{2}}{2 G}\left(1+\frac{c}{u}\left(1-\frac{G c}{\delta(\delta u+c) H}\right)\right)^{2}\right)\right) .
\end{aligned}
$$

In fact,

$$
\begin{gathered}
\operatorname{var}\left(Z_{t}\right)+\operatorname{var}\left(Z_{s}\right)-2 \operatorname{cov}\left(Z_{t}, Z_{s}\right)=g(s)+g(t)-2 h(s), \\
\frac{g(t)+g(s)-2 h(s)}{g(s) g(t)-h^{2}(s)}=\frac{1}{g(t)}+\frac{(h(t)-h(s))^{2}}{\left(g(s) g(t)-h^{2}(s)\right) g(t)} .
\end{gathered}
$$

So,

$$
\begin{aligned}
\exp ( & \left.-\frac{u^{2}}{2 \sigma^{2}}\left(\operatorname{var}\left(Z_{t}\right)+\operatorname{var}\left(Z_{s}\right)-2 \operatorname{cov}\left(Z_{t}, Z_{s}\right)\right)\right) \\
& \leq C_{5} \exp \left(-\frac{u^{2}}{2 g(t)}\right) \\
& \leq C_{6} \exp \left(-\frac{u^{2}}{2 G}\left(1+\frac{c}{u}\left(1-\frac{G c}{\delta(\delta u+c) H}\right)\right)^{2}\right) .
\end{aligned}
$$

From (2.6), (2.12), and (2.13), we finally obtain

$$
\begin{aligned}
& \mathrm{E}\left(N_{u}([\alpha, \beta])\left(N_{u}([\alpha, \beta])-1\right)\right) \\
& \quad=o\left(\log \left(\frac{(\delta u+c) H}{G c}\right) \exp \left(-\frac{u^{2}}{2 G}\left(1+\frac{c}{u}\left(1-\frac{G c}{\delta(\delta u+c) H}\right)\right)^{2}\right)\right) .
\end{aligned}
$$

Hence, the proof of Theorem 1.1 is completed.

\section{Acknowledgements}

The authors are grateful to an anonymous referee for comments and suggestions which led to the present improved version of the manuscript. The authors would also like to express their gratitude to Professor S. G. Kobelkov for his useful discussions and comments. This research was supported by the National Natural Science Foundation of China and the Ministry of Education of China.

\section{References}

[1] DȩBicKi, K. (2002). Ruin probability for Gaussian integrated processes. Stoch. Process. Appl. 98, 151-174.

[2] Dickson, D. C. M. And Egídio dos Reis, A. D. (1994). Ruin problems and dual events. Insurance Math. Econom. 14, 51-60.

[3] Dufresne, F. And Gerber, H. U. (1988). The probability and severity of ruin for combinations of exponential claim amount distributions and their translations. Insurance Math. Econom. 7, 75-80.

[4] Freidlin, M. I. And Wentzell, A. D. (1984). Random Perturbations of Dynamical Systems. Springer, New York.

[5] Hüsler, J. And Piterbarg, V. (1999). Extremes of a certain class of Gaussian processes. Stoch. Process. Appl. 83, 257-271.

[6] Hüsler, J. ANd Piterbarg, V. (2004). On the ruin probability for physical fractional Brownian motion. Stoch. Process. Appl. 113, 315-332. 
[7] Kobelkov, S. G. (2005). The ruin problem for the stationary Gaussian process. Stoch. Process. Appl. 49, 155-163.

[8] Piterbarg, V. I. (1996). Asymptotic Methods in the Theory of Gaussian Proceses and Fields (Transl. Math. Monogr. 148). American Mathematical Society, Providence, RI.

[9] Piterbarg, V. I. (1996). The Rice method for Gaussian random fields. Fundam. Prikl. Mat. 2, 187-204 (in Russian).

[10] Rolski, T., Schmidli, H., Schmidt, V. And Teugels, J. (1999). Stochastic Processes for Insurance and Finance. John Wiley, New York. 